\title{
Recurrent Laryngopyocele: CT-Guided Hookwire Localization for Re-Excision Surgery
}

\author{
A.R. Gafton, S.M. Cohen, J.D. Eastwood, M.K. Dang, and J.K. Hoang
}

\begin{abstract}
SUMMARY: Laryngopyocele recurrence after initial surgical resection is a very rare occurrence. We present a case of recurrent laryngopyocele in which CT fluoroscopy-guided hookwire placement was used to facilitate resection. In this article, we illustrate the imaging findings of laryngopyocele, review the approach to management, and describe the CT fluoroscopy-guided hookwire placement procedure.
\end{abstract}

aryngopyoceles are rare infectious complications of laryngoceles. The definitive treatment is surgical resection, performed after resolution of the acute infectious symptoms. We present a rare case of recurrent laryngopyocele after initial surgery, in which re-excision surgery was facilitated by CT fluoroscopy-guided hookwire placement.

\section{TECHNIQUE}

\section{Subject}

A 38-year-old man presented to the emergency department with a 1-week history of sore throat and productive cough and a 3-day history of fever, dysphagia, and odynophagia. Contrast-enhanced neck CT (Fig 1A, - B) demonstrated a large peripherally enhancing fluid collection with an air-fluid level centered in the right supraglottic larynx, bulging through the thyrohyoid membrane, compatible with a laryngopyocele.

Direct laryngoscopy showed edema and mucopurulence of the right piriform sinus, and epiglottic and false vocal cord edema narrowing the airway. The patient underwent fiberoptic intubation, endoscopic incision of right laryngeal ventricle, and drainage of purulent material. He was placed on broad-spectrum intravenous antibiotics and steroids. After a 2-day stay in the intensive

\footnotetext{
Received April 22, 2011; accepted after revision June 29.

From the Departments of Radiology (A.R.G., J.D.E., M.K.D., J.K.H.), Otolaryngology, Head and Neck Surgery (S.M.C.), and Radiation Oncology (J.K.H.), Duke University Hospital, Durham, North Carolina.

Paper previously presented at: Annual Meeting of the American Society of Neuroradiology, June 4-9, 2011; Seattle, Washington.

Please address correspondence to Jenny K. Hoang, MD, Department of Radiology, Duke University Hospital, Box 3808, Erwin Rd, Durham NC, 27710; e-mail: jennykh@gmail.com

http://dx.doi.org/10.3174/ajnr.A2810
}

care unit for airway support, the patient improved clinically and was discharged for outpatient follow-up.

Neck CT performed 2 months later showed a residual mixed laryngocele (Fig $1 C,-D$ ). Elective surgical resection of the laryngocele was performed.

Five months after surgery, the patient returned with recurrent symptoms, similar to those in his initial presentation. Neck CT demonstrated a recurrent right laryngopyocele. After treatment of the acute infection, elective laryngocele excision was again contemplated. It was thought that the external laryngocele wall had been incompletely resected due to scarring related to previous infection. Radiology was sought for preoperative imaging guidance.

\section{CT Fluoroscopy-Guided Hookwire Placement Technique}

On the morning of surgery, the patient presented for preoperative imaging-guided localization of the laryngocele wall. The procedure required a 20-ga Kopans spring hook localization needle kit (Cook, Bloomington, Indiana), which includes an introducer needle and a hookwire (Fig 2).

After providing informed consent, the patient was placed supine on the CT table, with his head slightly turned to the left. A skin marker was placed on the right side of the neck. A limited noncontrast CT scan was obtained through the area of interest for planning the needle approach (Fig $3 A$ ). The selected path avoided vascular structures and was favorable to the operative approach. After we marked the skin entry site, the right neck was prepared, draped, and locally anesthetized. With intermittent CT fluoroscopic guidance, the introducer needle was advanced to the lateral laryngocele wall (Fig 3B). Subsequently, the hookwire was inserted through the needle until the black marker on the hookwire was at the needle hub (indicating that the wire tip reached the needle tip). While holding the hookwire in place, we slowly with- 

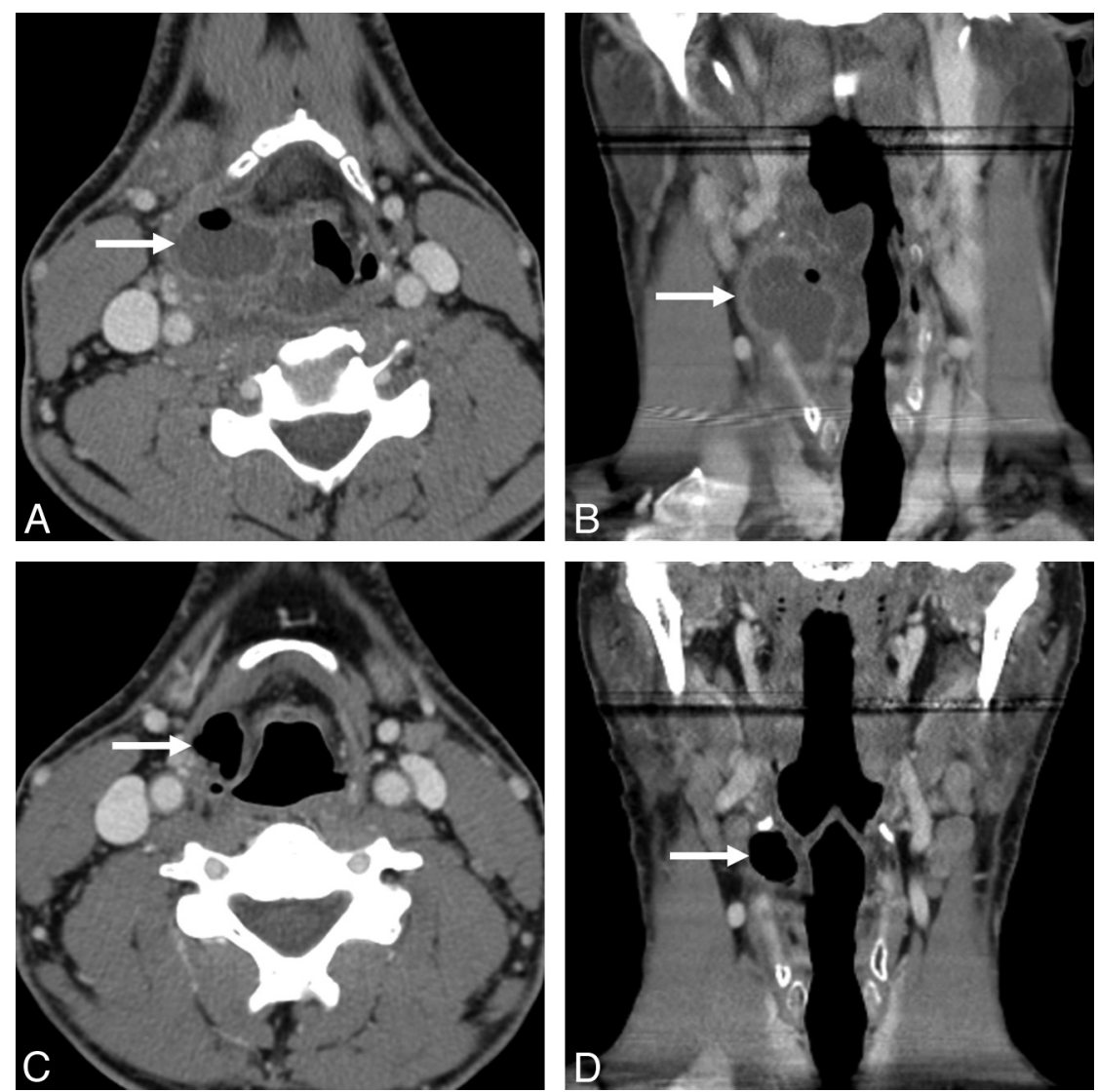

FIG 1. Thirty-eight-year-old man with 1-week history of sore throat and productive cough and 3-day history of fever, dysphagia, odynophagia, and inability to tolerate liquids and solids. $A$ and $B$, Contrast-enhanced axial $(A)$ and coronal $(B)$ neck CT images demonstrated a peripherally enhancing fluid collection with an air-fluid level in the right paraglottic space, bulging through the thyrohyoid membrane (arrows), consistent with a laryngopyocele. There was surrounding epiglottic and posterior pharyngeal wall edema with narrowing of the airway. $C$ and $D, A x i a l(C)$ and coronal $(D)$ neck CT images performed 2 months after the acute presentation demonstrated interval resolution of the peripherally enhancing fluid collection and a residual mixed right laryngocele (arrows), protruding through the thyrohyoid membrane.

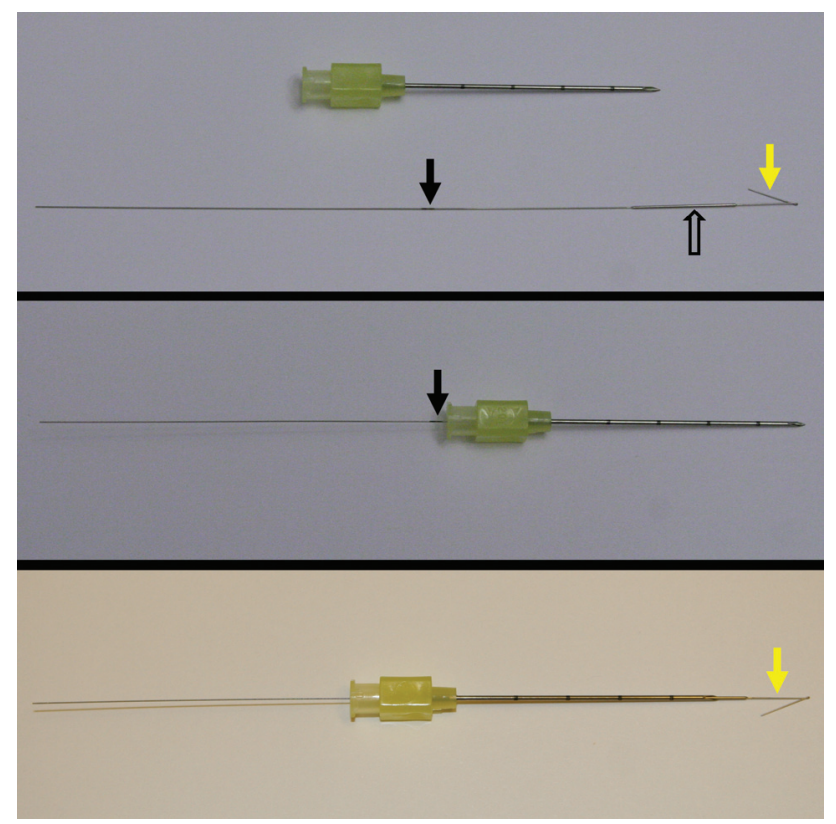

FIG 2. Kopans needle and hookwire localization kit (upper) with hookwire tip at the needle tip (middle) and the hookwire deployed through the needle (lower). When the black marker on the wire (black arrows) is at the level of the needle hub (middle image), the tip of the wire is at the tip of the needle. The distal $1 \mathrm{~cm}$ of the wire has a curved hook (yellow arrows), which allows the wire to be advanced in the lumen of the introducer needle (middle image) and then to spring open when the needle is withdrawn $>1 \mathrm{~cm}$ (lower image). The thicker component on the distal wire (open arrow in upper image) is just proximal to the hook and allows the surgeon to anticipate the depth to the tip. 

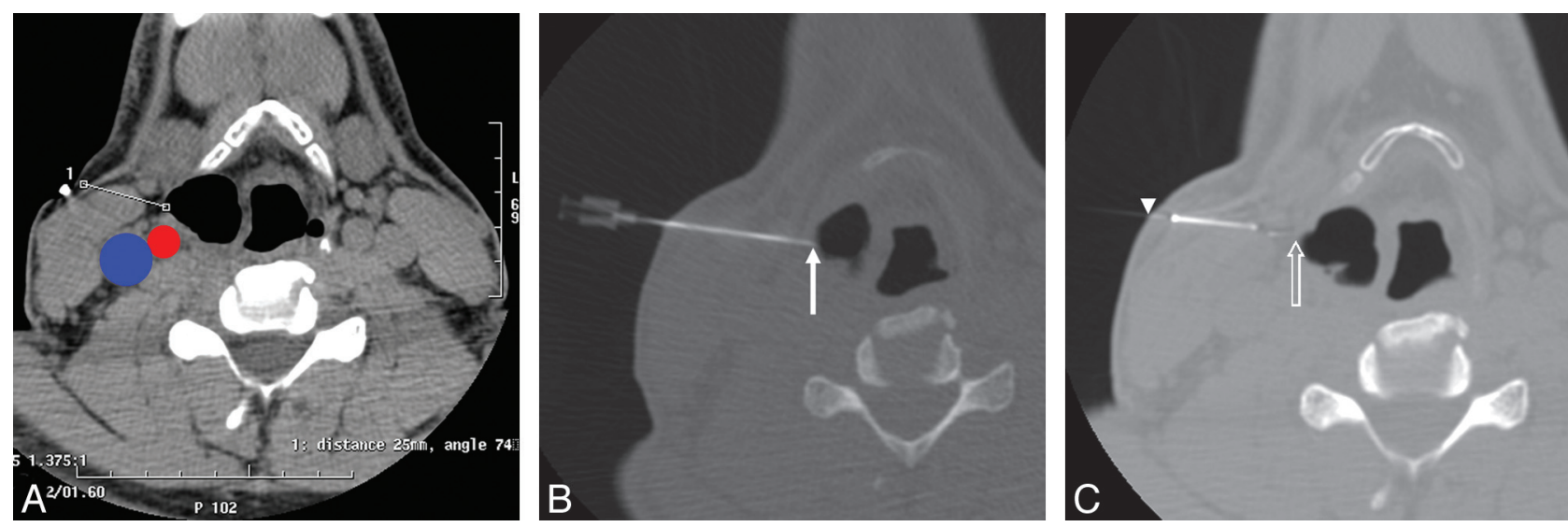

FIG 3. Hookwire placement technique. A, Preliminary noncontrast axial CT image was obtained for planning the needle approach (line in $A$ ) and measuring the distance between the skin-entry site and the desired needle-tip position at the level of the lateral laryngocele wall. The needle path should avoid major vascular structures: the carotid artery (red) and internal jugular vein (blue). $B$ and $C$, Axial $C T$ images during (B) and immediately following $(C)$ the needle-hookwire placement demonstrate the tip of the Kopans needle (arrow in $B$ ) and the tip of the hookwire (open arrow in C) at the level of the lateral laryngocele wall. Note the wire protruding through the skin (arrowhead in C).

drew the needle over the wire, causing the hook to spring open to anchor the wire in the surrounding tissues. The final position of the hookwire was confirmed with a limited CT scan (Fig 3C). The wire segment external to the skin was bent loosely and taped to prevent displacement. The patient was advised to avoid extreme ranges of neck movement and was transferred to the operating room.

\section{Outcome}

Two hours after hookwire placement, the patient underwent surgery. The skin incision was made anterior to the hookwire entry site, to minimize the risk of cutting or displacing the wire. The hookwire was identified in the surgical bed, and careful dissection was carried along the hookwire to the tip to identify the lateral laryngocele wall, which was embedded in scar tissue. Once the wall was identified, the hookwire was removed by cauterization. Subsequently, the entire laryngocele wall was dissected and resected successfully. The pathology showed a ciliated respiratory epithelial-lined cyst, consistent with a laryngocele. At his 6-month postsurgical follow-up, the patient was asymptomatic and was discharged from the clinic.

\section{DISCUSSION}

A laryngocele is an abnormal dilation of the anterior laryngeal ventricle, which extends superiorly between the false vocal cord and the inner aspect of the thyroid cartilage. Laryngopyoceles are rare infectious complications of laryngoceles. ${ }^{1}$ This technical note describes a case of recurrent laryngopyocele in which CT-guided hookwire localization enabled complete surgical resection.

Laryngopyoceles may present with life-threatening airway obstruction, palpable neck mass, hoarseness, dyspnea, dysphagia, odynophagia, and fever. ${ }^{2}$ In the acute phase, laryngopyocele management includes airway support, drainage, antibiotics, and steroids. ${ }^{3}$ After resolution of the acute infection, surgical resection of the laryngocele is performed electively to prevent recurrent infection. The recurrence rate of laryngocele after initial resection is low and largely depends on the surgical approach and experience of the surgeon. ${ }^{4}$ A potential cause for recurrence is incomplete laryngocele wall resection due to scarring related to prior infection. In our case, this was anticipated, so repeat surgery was performed after CT-guided hookwire localization of the laryngocele wall.

Hookwire localization is intended to help the surgeon find the lesion during surgery, thus allowing minimally invasive surgery. It is most commonly used for preoperative localization of occult breast lesions under mammography or sonography, but hookwire placement has also been performed for other body regions. ${ }^{5} \mathrm{Sev}$ eral case reports and small case series described preoperative CTguided hookwire placement for sclerotic rib lesions, paraspinal and popliteal fossa soft-tissue lesions, intramuscular hemangiomas, lung lesions, small lymph nodes, peritoneal cysts, appendicoliths, and peripheral nerve lesions. ${ }^{6-12}$ Within the head and neck region, hookwire placement has been used for preoperative localization of deep infratemporal fossa foreign bodies, brachial plexus neurofibromas, and deep and impalpable cervical lymph nodes. ${ }^{10,13,14}$ In our patient, hookwire placement was performed to identify the laryngocele wall to ensure complete resection.

Although the hookwire-placement procedure and surgical excision were uncomplicated in our case, there are 2 potential pitfalls. First, the hookwire can migrate from the intended position, before or during surgery. The "fishhook" configuration of the wire tip prevents backward movement, but deep migration is possible. This can be reduced by bending the hookwire $90^{\circ}$ at the skin surface to prevent inward movement and advising the patient not to move the localized body part. ${ }^{15}$ Second, transection of the wire during surgery with hook retention has been reported. ${ }^{16}$ For this reason, our surgeon made a skin incision separate from the hookwire skin entry site. This problem can also be prevented by careful dissection along the path of the wire and accounting for the entire length of wire if the wire is fragmented.

In summary, we presented a case of a laryngopyocele, which recurred after initial surgical resection. CT-guided hookwire placement for preoperative localization of the impalpable external laryngocele wall allowed the surgeon to accurately identify and completely resect the laryngocele and reduced the need for larger exploration and re-excision.

AJNR Am J Neuroradiol 34:E39-E42 Apr 2013 www.ajnr.org 
Disclosures: Seth Cohen-UNRELATED: Grants/Grants Pending: research funding from the American Academy of Otolaryngology-Head and Neck Surgery.

\section{REFERENCES}

1. Cassano L, Lombardo P, Ragona RM, et al. Laryngopyocele: three new clinical cases and review of the literature. Eur Arch Otorhinolaryngol 2000;257:507-11

2. Nazaroglu H, Özates M, Uyar A, et al. Laryngopyocele: signs on computed tomography. Eur J Radiol 2000;33:63-65

3. Papila İ, Acioğlu E, Karaman E, et al. Laryngeal chondroma presenting as a laryngopyocele. Eur Arch Otorhinolaryngol 2005;262:473-76

4. Vengerovich G, McCoul ED, Burstein DH, et al. Excision of laryngocele via transcervical midline approach. Laryngoscope 2010; 120(suppl 4):S189

5. Kopans DB, DeLuca S. A modified needle-hookwire technique to simplify preoperative localization of occult breast lesions. Radiology 1980;134:781

6. Slotty PJ, Kröpil P, Klingenhöfer M, et al. Preoperative localization of spinal and peripheral pathologies for surgery by computed tomography-guided placement of a specialized needle system. Neurosurgery 2010;66:784-87

7. Morrison WB, Sanders TG, Parsons TW, et al. Preoperative CTguided hookwire needle localization of musculoskeletal lesions. AJR Am J Roentgenol 2001;176:1531-33

8. Quinn PS, Sieunarine K, Lawrence-Brown M, et al. Intramuscular haemangiomas: hookwire localization prior to surgical excisionreport of four cases. ANZ J Surg 2001;71:62-66

9. Thaete FL, Peterson MS, Plunkett MB, et al. Computed tomographyguided wire localization of pulmonary lesions before thoracoscopic resection: results in 101 cases. J Thorac Imaging 1999;14:90-98

10. Finch I. Preoperative CT-guided percutaneous localization of small masses with a Kopans needle. AJR Am J Roentgenol 1991;157:179-80

11. Kagalwala DZ, Shankar S, Zota V, et al. Preoperative computed tomography-guided hookwire needle localization of a peritoneal multilocular inclusion cyst. J Comput Assist Tomogr 2005;29:602-03

12. Lossef SV. CT-guided Kopans hookwire placement for preoperative localization of an appendicolith. AJR Am J Roentgenol 2005; 185:81-83

13. Hoang JK, DeJesus R, Eastwood JD, et al. CT-guided hookwire placement: a technical innovation for preoperative localization of nonpalpable cervical lymph nodes. AJNR Am J Neuroradiol 2012;33: E104-06

14. Shemen LJ, Schechter LS, Godfrey N. Needle-wire localization of an infratemporal fossa foreign body using computed tomography. Arch Otolaryngol Head Neck Surg 1992;118:1337-39

15. Bronstein AD, Kilcoyne RF, Moe RE. Complications of needle localization of foreign bodies and nonpalpable breast lesions. Arch Surg 1988;123:775-79

16. Guermazi A, Miaux $\mathrm{Y}$, Genant $\mathrm{HK}$, et al. Be sure to insertappropriately and safely-a hookwire! AJR Am J Roentgenol 2002; 178:764-65 